\title{
Activité antifongique de quelques plantes de la flore ivoirienne
}

\author{
Fézan H. Tra BI ${ }^{1 *}$, N'Guessan F. Kouamé ${ }^{2}$, Anne $\mathrm{FAVEL}^{3}$ \& Karim Fallague ${ }^{3}$. \\ ${ }^{1}$ Université d'Abobo-Adjamé Abidjan, UFR SN, 02 BP 801 Abidjan 02 (Côte d'Ivoire) \\ ${ }^{2}$ Université de Cocody Abidjan, UFR Biosciences, 22 BP 582 Abidjan 22 (Côte d'Ivoire) \\ ${ }^{3}$ Faculté de Pharmacie, Laboratoire de Botanique, Cryptogamie et Biologie cellulaire, Université de la Méditerranée Aix-Marseille II, 27 \\ Boulevard Jean Moulin 13385 Marseille Cedex 5 (France). \\ *Auteur pour les correspondances (Email : trabi_fezan@hotmail.com) \\ Reçu le 20-02-2006, accepté le 12-03-2007.
}

\section{Résumé}

Six espèces de plantes de la flore ivoirienne ont été utilisées pour la recherche de leur activité antifongique. Ce sont : Borreria latifolia, Borreria verticillata, Erigeron floribundus, Euphorbia hirta, Turraea heterophylla et Vernonia colorata. Les extraits dichlorométhane, méthanolique et aqueux, réalisés à partir des poudres de feuilles ou de rameaux feuillés de chaque espèce, ont permis d'évaluer cette activité sur les levures ( 9 souches de référence et 10 souches cliniques). Seul l'extrait dichlorométhane de Erigeron floribundus a présenté une activité inhibitrice sur les champignons avec une Concentration Minimale Fongicide (CMF) située entre $0,5 \mathrm{mg} / \mathrm{ml}$ et $1 \mathrm{mg} / \mathrm{ml}$.

Mots clefs: Plantes; Activité antifongique; Extraits bruts ; Flore ivoirienne.

\begin{abstract}
Antifungal activity of plants from flora of Côte d'Ivoire.

Six plant species from flora of Côte d'Ivoire were screened for antifungal activities. These plant species are: Borreria latifolia, Borreria verticillata, Erigeron floribundus, Euphorbia hirta, Turraea heterophylla and Vernonia colorata. The dichloromethane, methanol and aqueous extracts from the powered leave sample of each species were tested using yeast (9 reference strains and 10 clinical strains). Only dichloromethane extract of Erigeron floribundus showed an activity. The MFC was between $0.5 \mathrm{mg} / \mathrm{ml}$ and $1 \mathrm{mg} / \mathrm{ml}$.
\end{abstract}

Keywords: Plants ; Antifungal activity ; Crude extracts ; Flora of Côte d'Ivoire

\section{Introduction}

Les populations des forêts classées du HautSassandra (Centre-Ouest) et de Scio (Ouest) en Côte d'Ivoire, vivent dans les zones de culture de produits agricoles : café, cacao et vivriers. A cause de leur éloignement des centres médicaux, elles exploitent abondamment les plantes médicinales dans le traitement de maladies ne nécessitant pas dans l'immédiat, une évacuation dans un centre hospitalier. Ainsi, Borreria latifolia (Rubiaceae), Borreria verticillata (Rubiaceae),
Erigeron floribundus (Asteraceae), Euphorbia hirta (Euphorbiaceae), Turraea heterophylla (Méliaceae) et Vernonia colorata (Asteraceae), sont elles employées pour leurs vertus antifongiques ( $T r a \mathrm{Bi}, 1997$ ).

Après des enquêtes ethnobotaniques réalisées auprès des populations rurales et des couches sociales démunies de certaines grandes agglomérations à forte concentration humaine dont la ville d'Abidjan, la capitale économique de la Côte d'Ivoire, il ressort que ces plantes sont 
également recherchées pour le traitement des maladies de la peau et du cuir chevelu, principalement les mycoses superficielles.

Les mycoses superficielles sont des infections à champignons microscopiques. Ce sont, essentiellement, des dermatophytoses dues à des dermatophytes ou des candidoses causées par des levures. Les candidoses qui constituent les plus fréquentes des infections à levures sont naturellement présentes sur la peau et dans l'intestin. Sur la peau, elles se rencontrent fréquemment dans les plis et aux fesses, surtout chez les jeunes enfants. Candida albicans et Candida parapsilosis en sont les principaux responsables. En effet, selon Hilmarsdottir et Datry (1993), Koenig (1995), Midgley et al. (1998), ces deux levures sont responsables des mycoses cutanées et des onyxis. Selon Midgley et al. (1998), l'infection des espaces interdigitaux des doigts ou des orteils par Candida est plus fréquente sous les climats chauds, surtout chez les militaires.

La valorisation des plantes médicinales de la flore ivoirienne nous a conduit à nous intéresser à l'activité antifongique des extraits des poudres de plantes sur les levures. Pour ce faire, nous avons suivi, au laboratoire, in vitro, l'activité de trois types d'extraits bruts de feuilles ou de rameaux feuillés de plantes sur les souches de référence et les souches cliniques de levures.

\section{Matériel et méthodes}

\subsection{Matériel}

Il est composé essentiellement de poudre de feuilles ou de rameaux feuillés (Tableau 1 ) de Borreria latifolia (J.B. Aublet) K. Schumann ou Spermacoce latifolia Aubl. (Rubiaceae), Borreria verticillata (L.) G. F. Mey ou Spermacoce verticillata L. (Rubiaceae), Erigeron floribundus (Kunth) Sch. Bip. ou Conyza sumatrensis (Retz.) E. H. Walker (Asteraceae), Euphorbia hirta L. (Euphorbiaceae), Turraea heterophylla J. Sm. (Meliaceae) et Vernonia colorata (Willd.) Drake. (Asteraceae). Les parties aériennes des plantes ont été prélevées et identifiées au Centre National de Floristique du Jardin Botanique de I'Université de Cocody, Abidjan. Pour nommer les différentes espèces, nous avons adopté la nomenclature de Hutchinson et Dalziel (1954) révisée par Lebrun et Stork (1992; 1997).
Tableau 1 : Nature des organes prélevés pour la réalisation des poudres de plantes

\begin{tabular}{ll}
\hline Espèces végétales & Organes prélevés \\
\hline Borreria latifolia & Rameaux feuillés \\
Borreria verticillata & Rameaux feuillés \\
Erigeron floribundus & Rameaux feuillés \\
Euphorbia hirta & Rameaux feuillés \\
Turraea heterophylla & Feuilles \\
Vernonia colorata & Feuilles \\
\hline
\end{tabular}

Le matériel comprend, en outre, des souches de référence de levures (Candida albicans ATCC 90029, Candida albicans ATCC 38248, Candida albicans Y 0109, Candida tropicalis IP 1275-81, Candida parapsilosis ATCC 22019, Candida glabrata ATCC 90030, Candida kefyr Y 0601, Candida krusei ATCC 6258, Candida lusitaniae CBS 6936) et des souches cliniques de levures (Candida tropicalis anus, Candida krusei HB 7, Candida glabrata 13668197 (19G), Candida glabrata HB 10 (57G), Candida parapsilosis Bonjardi, Candida norvegensis PSP, Candida albicans 76A, Candida albicans 78A, Candida lusitaniae 215L, Candida lusitaniae 6-103 D 54L).

Les souches de référence proviennent des collections enregistrées à l'American Type Culture Collection (ATCC), à l'Institut Pasteur (IP) de Paris, au Centraalbureau voor Schimmelcultures (CBS) des Pays Bas et chez le Laboratoire Pfizer (Y). Les souches cliniques sont des prélèvements humains provenant des hôpitaux et des laboratoires d'analyses médicales de Marseille et de certaines villes de France.

\subsection{Méthodes d'études}

\subsubsection{Obtention des extraits bruts}

Pour obtenir les extraits bruts, 3 solvants ont été utilisés : le dichlorométhane RS (Carlo Erba), le méthanol RE (Carlo Erba) $80 \%$ et l'eau distillée. L'extrait dicholorométhane (DCM) est obtenu après macération dans ce solvant, de $100 \mathrm{~g}$ de poudre pendant 15 heures en moyenne dans une colonne en verre de $60 \mathrm{~cm}$ de long et $4,8 \mathrm{~cm}$ de diamètre. Une lixiviation suivie d'un passage au rotavapor a permis d'obtenir, après séchage dans un verre de montre, sous hotte, l'extrait dichlorométhane. La poudre résiduelle a été à son tour macérée dans du méthanol à $80 \%$. Après lixiviation suivie de séchage au rotavapor, il reste une solution aqueuse qui, lyophilisée, produit l'extrait méthanolique $80 \%$ (MeOH80). Pour l'extrait aqueux (Aq), $10 \mathrm{~g}$ de poudre ont été macérés dans de l'eau distillée portée à ébullition. Le filtrat lyophilisé a permis d'obtenir cet extrait. 


\subsubsection{Tests antifongiques}

Pour réaliser les tests antifongiques, nous avons procédé par la méthode de dilution en milieu solide utilisée par Favel et al. (1994). Elle consiste à incorporer l'extrait brut dans un milieu gélosé, une solution à $2 \%$ de Bacto agar (DIFCO) préalablement portée à $60^{\circ} \mathrm{C}$ avant solidification. L'extrait à tester est pesé dans un tube à hémolyse et solubilisé dans une solution de Dimethyl Sulfoxide (DMSO-RPE Carlo Erba). La concentration finale en DMSO dans le milieu de culture ne doit pas être supérieure à $2 \%$. Chaque extrait brut est testé aux concentrations de 1 et 2 $\mathrm{mg} / \mathrm{ml}$ de milieu.

Pour les différentes souches à tester, nous avons réalisé une suspension correspondant à une opacité de 0.5 à 1 sur l'échelle de Mc. Farland dans $3 \mathrm{ml}$ de sérum physiologique ( $\mathrm{NaCl} 0.9 \%$ ). Les souches sont ensemencées sous forme de spots d'environ $10 \mu \mathrm{l}$ à la surface de la gélose dans une boîte de Pétri de 90 $\mathrm{mm}$ de diamètre préalablement marquée. La lecture se fait après $48 \mathrm{~h}$ d'incubation à $35^{\circ} \mathrm{C}$. Si les colonies se développent normalement, le produit n'a pas d'activité antifongique. Inversement, si la croissance fongique est inhibée, nous en déduisons que le produit a une activité antifongique.

Lorsque l'extrait présente une activité antifongique, les concentrations inférieures $(0,5 \mathrm{mg} / \mathrm{ml} ; 0,25$ $\mathrm{mg} / \mathrm{ml} ; 0,125 \mathrm{mg} / \mathrm{ml} ; 0,06 \mathrm{mg} / \mathrm{ml})$ sont testées afin de déterminer la Concentration Minimale Fongicide (CMF) qui correspond à la plus faible concentration à laquelle on observe la mort des levures.

\section{Résultats et Discussion}

La quantité d'extraits bruts obtenue (pour $100 \mathrm{~g}$ de poudre avec les solvants organiques et $10 \mathrm{~g}$ de poudre pour l'eau distillée) varie suivant le solvant (Tableau 2). Le méthanol $80 \%$ et l'eau distillée sont les solvants qui permettent d'obtenir plus d'extraits bruts. B. latifolia, E. floribundus et $V$. colorata sont les plantes qui donnent suffisamment d'extraits bruts avec ces solvants.

Tous les extraits de B. latifolia, B. verticillata, E. hirta, T. heterophylla, V.colorata et les extraits aqueux et méthanolique $80 \%$ de $E$. floribundus n'ont présenté aucune activité antifongique à $2 \mathrm{mg} /$ $\mathrm{ml}$. Seul l'extrait dichlorométhane de E. floribundus a présenté en dessous de cette concentration, une activité inhibitrice sur les levures. Cette activité a été observée sur 6 souches de référence et 6 souches cliniques de levures. La CMF se situe entre $0,5 \mathrm{mg} / \mathrm{ml}$ et $1 \mathrm{mg} / \mathrm{ml}$ (Tableaux $3 \& 4$ ).
L'analyse des résultats fait apparaître que les souches de référence et les souches cliniques de levures ont des sensibilités presque identiques à l'activité de l'extrait dichlorométhane de E. floribundus, puisque 6 des 9 souches utilisées présentent une CMF comprise entre 1 $\mathrm{mg} / \mathrm{ml}$ et $0,5 \mathrm{mg} / \mathrm{ml}$. Candida albicans, $C$. tropicalis, C. lusitaniae et $C$. parapsilosis sont les souches les plus sensibles. C. glabrata, C. kefyr et $C$. krusei qui ont présenté des CMF supérieures ou égales à $2 \mathrm{mg} / \mathrm{ml}$ sont les souches les moins sensibles à l'extrait brut.

Les différentes CMF observées permettent de suggérer que l'extrait dichlorméthane de $E$. floribundus présente, à $2 \mathrm{mg} / \mathrm{ml}$, une activité antifongique intéressante sur les souches de levures testées. Les extraits méthanolique $80 \%$ et aqueux ont été inactifs. L'inactivité dans l'extrait alcoolique de E. floribundus confirme les travaux de Vlietinck et al. (1995), qui ont montré que l'extrait l'éthanolique $80 \%$ de cette espèce n'avait aucune activité sur $C$. albicans. Mieux, l'activité antifongique de cet extrait n'était perceptible qu'à $500 \mathrm{mg} / \mathrm{ml}$ sur les champignons dermatophytes Microsporum canis et Trichophyton mentagrophytes qui sont également responsables de certaines affections fongiques cutanées. Hamill et al. (2003) n'ont observé qu'une activité non significative de $E$. floribundus sur $C$. albicans. La présence d'une activité antifongique dans l'extrait dichlorométhane de E. floribundus et l'absence de cette activité dans l'extrait méthanolique $80 \%$ et l'extrait aqueux pourraient s'expliquer par l'existence dans chaque type d'extrait de composés différents. En effet, ces solvants sont de polarités différentes si bien qu'ils entraînent, au cours des opérations d'extraction, des composés différents.

Asongalem et al. (2004) ont mentionné la présence dans les feuilles de E. floribundus, de saponines, de flavonoïdes, de glycosides, d'alcaloïdes, d'huiles essentielles, de tanins et de phénols. Les travaux de Ebi et Kamalu (2001) ont montré l'activité antifongique des extraits dichlorométhane, méthanolique, ethanolique de Mitracarpus scaber (Rubiaceae), sur Aspergillus flavus et $C$. albicans. Ils ont indiqué la présence d'alcaloïdes et de saponines dans ces extraits et ont attribué l'activité des extraits aux saponines qui interagiraient avec les stérols, les protéines et les phospholipides des membranes cellulaires des champignons. Tatsadjieu et al. (2003) ont montré par la suite, l'action des huiles essentielles extraites de Xylopia aethiopica, Monodora myristica, Zanthoxylum xanthoxyloides et Zanthoxylum leprieurii sur 
Aspergillus flavus.

On pourrait à partir de ces résultats, supposer que l'activité de l'extrait dichlorométhane de $E$. floribundus serait liée à la présence dans cet extrait, d'huiles essentielles ou de saponines. L'activité antifongique de l'extrait dichlorométhane de $E$. floribundus observée au cours de cette étude explique, en partie, l'utilisation de la plante par les populations rurales et urbaines ivoiriennes, dans le traitement des affections cutanées dont certaines levures sont responsables (Tra Bi, 1997). Cette utilisation est d'autant plus justifiée que les tests in vitro donnent des résultats intéressants à de faibles CMF $(0,5$ à $1 \mathrm{mg} / \mathrm{ml})$.

Tableau 2 : Masse d'extraits bruts produits par espèce et par solvant

\begin{tabular}{llcc}
\hline & \multicolumn{3}{c}{ Masses des extraits bruts $(g)$} \\
\cline { 2 - 4 } & Dichlorométhane & Méthanol 80 & Eau \\
\hline Borreria latifolia & 3,95 & 11,80 & 1,11 \\
Borreria verticillata & 3,43 & 8,10 & 0,48 \\
Erigeron floribundus & 4,20 & 15,05 & 1,86 \\
Euphorbia hirta & 2,88 & 12,78 & 0,42 \\
Turraea heterophylla & 3,87 & 5,16 & 1,42 \\
Vernonia colorata & 4,68 & 18 & 1,95 \\
\hline
\end{tabular}

Tableau 3 : Activité antifongique des extraits bruts de Erigeron floribundus, Borreria latifolia, Borreria verticillata, Euphorbia hirta, Turraea heterophylla et Vernonia colorata sur les souches de référence de levures.

\begin{tabular}{|c|c|c|c|c|c|c|c|c|c|}
\hline \multicolumn{10}{|c|}{ Concentration Minimale Fongicide (mg/ml) } \\
\hline \multicolumn{10}{|c|}{ Erigeron floribundus } \\
\hline & $\begin{array}{c}\text { C. } \\
\text { albicans } \\
\text { ATCC } \\
90029\end{array}$ & $\begin{array}{c}\text { C. } \\
\text { albicans } \\
\text { ATCC } \\
38248\end{array}$ & $\begin{array}{c}\text { C. } \\
\text { albicans } \\
\text { Y } 0109\end{array}$ & $\begin{array}{c}\text { C. } \\
\text { tropicali } \\
\text { s } \\
\text { IP } 1275- \\
81\end{array}$ & $\begin{array}{c}\text { C. } \\
\text { parapsilo } \\
\text { sis } \\
\text { ATCC } \\
22019\end{array}$ & $\begin{array}{c}\text { C. } \\
\text { glabrata } \\
\text { ATCC } \\
90030\end{array}$ & $\begin{array}{c}\text { C. } \\
\text { kefyr } \\
\text { Y } 0601\end{array}$ & $\begin{array}{c}\text { C. } \\
\text { krusei } \\
\text { ATCC } \\
6258\end{array}$ & $\begin{array}{c}\text { C. } \\
\text { lusitani } \\
\text { ae } \\
\text { CBS } \\
6936\end{array}$ \\
\hline \multicolumn{10}{|l|}{ Extraits } \\
\hline DCM & 0,5 & 0,5 & 0,5 & 0,5 & 1 & 2 & 2 & $>2$ & 0,5 \\
\hline МeOH80 & $>2$ & $>2$ & $>2$ & $>2$ & $>2$ & $>2$ & $>2$ & $>2$ & $>2$ \\
\hline $\mathrm{Aq}$ & $>2$ & $>2$ & $>2$ & $>2$ & $>2$ & $>2$ & $>2$ & $>2$ & $>2$ \\
\hline \multicolumn{10}{|c|}{ Borreria latifolia } \\
\hline DCM & $>2$ & $>2$ & $>2$ & $>2$ & $>2$ & $>2$ & $>2$ & $>2$ & $>2$ \\
\hline МeOH80 & $>2$ & $>2$ & $>2$ & $>2$ & $>2$ & $>2$ & $>2$ & $>2$ & $>2$ \\
\hline $\mathrm{Aq}$ & $>2$ & $>2$ & $>2$ & $>2$ & $>2$ & $>2$ & $>2$ & $>2$ & $>2$ \\
\hline \multicolumn{10}{|c|}{ Borreria verticillata } \\
\hline DCM & $>2$ & $>2$ & $>2$ & $>2$ & $>2$ & $>2$ & $>2$ & $>2$ & $>2$ \\
\hline МeOH80 & $>2$ & $>2$ & $>2$ & $>2$ & $>2$ & $>2$ & $>2$ & $>2$ & $>2$ \\
\hline $\mathrm{Aq}$ & $>2$ & $>2$ & $>2$ & $>2$ & $>2$ & $>2$ & $>2$ & $>2$ & $>2$ \\
\hline \multicolumn{10}{|c|}{ Euphorbia hirta } \\
\hline DCM & $>2$ & $>2$ & $>2$ & $>2$ & $>2$ & $>2$ & $>2$ & $>2$ & $>2$ \\
\hline МeOH80 & $>2$ & $>2$ & $>2$ & $>2$ & $>2$ & $>2$ & $>2$ & $>2$ & $>2$ \\
\hline $\mathrm{Aq}$ & $>2$ & $>2$ & $>2$ & $>2$ & $>2$ & $>2$ & $>2$ & $>2$ & $>2$ \\
\hline \multicolumn{10}{|c|}{ Turraea heterophylla } \\
\hline DCM & $>2$ & $>2$ & $>2$ & $>2$ & $>2$ & $>2$ & $>2$ & $>2$ & $>2$ \\
\hline МeOH80 & $>2$ & $>2$ & $>2$ & $>2$ & $>2$ & $>2$ & $>2$ & $>2$ & $>2$ \\
\hline $\mathrm{Aq}$ & $>2$ & $>2$ & $>2$ & $>2$ & $>2$ & $>2$ & $>2$ & $>2$ & $>2$ \\
\hline \multicolumn{10}{|c|}{ Vernonia colorata } \\
\hline DCM & $>2$ & $>2$ & $>2$ & $>2$ & $>2$ & $>2$ & $>2$ & $>2$ & $>2$ \\
\hline МeOH80 & $>2$ & $>2$ & $>2$ & $>2$ & $>2$ & $>2$ & $>2$ & $>2$ & $>2$ \\
\hline $\mathrm{Aq}$ & $>2$ & $>2$ & $>2$ & $>2$ & $>2$ & $>2$ & $>2$ & $>2$ & $>2$ \\
\hline
\end{tabular}


Tableau 4 : Activité antifongique des extraits bruts de Erigeron floribundus, Borreria latifolia, Borreria verticillata, Euphorbia hirta, Turraea heterophylla et Vernonia colorata sur les souches cliniques de levures.

\begin{tabular}{|c|c|c|c|c|c|c|c|c|c|c|}
\hline \multicolumn{11}{|c|}{ Concentration Minimale Fongicide (mg/ml) } \\
\hline \multicolumn{11}{|c|}{ Erigeron floribundus } \\
\hline & $\begin{array}{c}\text { C. } \\
\text { tropicalis } \\
\text { anus }\end{array}$ & $\begin{array}{c}\text { C. } \\
\text { krusei } \\
\text { HB } 7\end{array}$ & $\begin{array}{c}C . \\
\text { glabrata } \\
136681 \\
(19 \mathrm{G})\end{array}$ & $\begin{array}{c}\text { C. } \\
\text { glabrata } \\
\text { HB 10 } \\
(57 \mathrm{G})\end{array}$ & $\begin{array}{c}\text { C. } \\
\text { parapsilo } \\
\text { sis } \\
\text { Bonjardi }\end{array}$ & $\begin{array}{c}\text { C. } \\
\text { norveg } \\
\text { ensis } \\
\text { PSP }\end{array}$ & $\begin{array}{c}\text { C. } \\
\text { albicans } \\
76 \mathrm{~A}\end{array}$ & $\begin{array}{c}\text { C. } \\
\text { albicans } \\
78 \mathrm{~A}\end{array}$ & $\begin{array}{c}C . \\
\text { lusitaniae } \\
215 \mathrm{~L}\end{array}$ & $\begin{array}{c}C . \\
\text { Iusitaniae } \\
\text { 6-103D } \\
\text { (54L) }\end{array}$ \\
\hline \multicolumn{11}{|l|}{ Extraits } \\
\hline $\mathrm{DCM}$ & 1 & $>2$ & $>2$ & $>2$ & 0,5 & 1 & 1 & 0,5 & 0,5 & $>2$ \\
\hline $\mathrm{MeOH} 80$ & $>2$ & $>2$ & $>2$ & $>2$ & $>2$ & $>2$ & $>2$ & $>2$ & $>2$ & $>2$ \\
\hline $\mathrm{Aq}$ & $>2$ & $>2$ & $>2$ & $>2$ & $>2$ & $>2$ & $>2$ & $>2$ & $>2$ & $>2$ \\
\hline \multicolumn{11}{|c|}{ Borreria latifolia } \\
\hline DCM & $>2$ & $>2$ & $>2$ & $>2$ & $>2$ & $>2$ & $>2$ & $>2$ & $>2$ & $>2$ \\
\hline МeOH80 & $>2$ & $>2$ & $>2$ & $>2$ & $>2$ & $>2$ & $>2$ & $>2$ & $>2$ & $>2$ \\
\hline $\mathrm{Aq}$ & $>2$ & $>2$ & $>2$ & $>2$ & $>2$ & $>2$ & $>2$ & $>2$ & $>2$ & $>2$ \\
\hline \multicolumn{11}{|c|}{ Borreria verticillata } \\
\hline DCM & $>2$ & $>2$ & $>2$ & $>2$ & $>2$ & $>2$ & $>2$ & $>2$ & $>2$ & $>2$ \\
\hline MeOH80 & $>2$ & $>2$ & $>2$ & $>2$ & $>2$ & $>2$ & $>2$ & $>2$ & $>2$ & $>2$ \\
\hline $\mathrm{Aq}$ & $>2$ & $>2$ & $>2$ & $>2$ & $>2$ & $>2$ & $>2$ & $>2$ & $>2$ & $>2$ \\
\hline \multicolumn{11}{|c|}{ Euphorbia hirta } \\
\hline DCM & $>2$ & $>2$ & $>2$ & $>2$ & $>2$ & $>2$ & $>2$ & $>2$ & $>2$ & $>2$ \\
\hline МeOH80 & $>2$ & $>2$ & $>2$ & $>2$ & $>2$ & $>2$ & $>2$ & $>2$ & $>2$ & $>2$ \\
\hline $\mathrm{Aq}$ & $>2$ & $>2$ & $>2$ & $>2$ & $>2$ & $>2$ & $>2$ & $>2$ & $>2$ & $>2$ \\
\hline \multicolumn{11}{|c|}{ Turraea heterophylla } \\
\hline DCM & $>2$ & $>2$ & $>2$ & $>2$ & $>2$ & $>2$ & $>2$ & $>2$ & $>2$ & $>2$ \\
\hline МeOH80 & $>2$ & $>2$ & $>2$ & $>2$ & $>2$ & $>2$ & $>2$ & $>2$ & $>2$ & $>2$ \\
\hline $\mathrm{Aq}$ & $>2$ & $>2$ & $>2$ & $>2$ & $>2$ & $>2$ & $>2$ & $>2$ & $>2$ & $>2$ \\
\hline \multicolumn{11}{|c|}{ Vernonia colorata } \\
\hline DCM & $>2$ & $>2$ & $>2$ & $>2$ & $>2$ & $>2$ & $>2$ & $>2$ & $>2$ & $>2$ \\
\hline $\mathrm{MeOH} 80$ & $>2$ & $>2$ & $>2$ & $>2$ & $>2$ & $>2$ & $>2$ & $>2$ & $>2$ & $>2$ \\
\hline $\mathrm{Aq}$ & $>2$ & $>2$ & $>2$ & $>2$ & $>2$ & $>2$ & $>2$ & $>2$ & $>2$ & $>2$ \\
\hline
\end{tabular}

\section{Conclusion}

L'extrait brut de E. floribundus a présenté une activité antifongique relativement élevée sur les souches de référence et les souches cliniques de levures. II présente un intérêt pratique dans la lutte contre les mycoses superficielles. Ce résultat va permettre de tester son activité sur les dermatophytes. Si l'activité est conservée et améliorée sur les dermatophytes, nous procéderons aux fractionnements de l'extrait brut, à la purification et à l'identification du ou des composés qui en sont responsables. Nous étudierons par la suite, le mode d'action de la molécule sur les organismes pathogènes.

\section{Références citées}

Asongalem E.A., Foyet H.S., Ngogang J., Folefoc G.N., Dimo T. \& Kamtchouing P., 2004. Analgesic and antiinflammatory activities of Erigeron floribundus. J. Ethnopharmacol. 91: 301-308.

Ebi G.C. \& Kamalu T.N., 2001. Phytochemical and antimicrobial properties of constituents of «Ogwu Odenigbo»', a popular Nigerian herbal medicine for typhoid fever. Phytother. Res. 15: 73-75.

Favel A., Steinmetz M.D. \& Regli P., 1994. In vitro antifungal activity of triterpenoid saponins. Planta Med. 60: 50-53.

Hamill F.A., Apio S., Mubiru N.K., Bukenya-Ziraba R., Mosango M., Maganyi O.W. \& Soejarto D.D., 2003. Traditional herbal drugs of Southern Uganda, II: literature analysis and antimicrobial assays. J. Ethnopharmacol. 84: 57-78.

Hilmarsdottir I. \& Datry A., 1993. Mycoses superficielles. In: Piérard G.E., Caumes C., Arrese Estrada J. \& Franchimont C., Eds. Dermatologie tropicale. DeBook ed. Bruxelles (Belgique): AUPELF, pp 632. 
Hutchinson J. \& Dalziel J.M., 1954. Flora of West Tropical Africa. London (England): Crown Agents for Oversea Government and Administratons Millbank; 828 pp.

Koenig H., 1995. Guide de mycologie médicale. Paris (France): Ellipse; 284 pp.

Lebrun J.-P. \& Stork A.L., 1992. Enumération des plantes à fleurs d'Afrique Tropicale: Chrysobalanaceae à Apiaceae. Génève (Suisse): CJB; 257 pp.

Lebrun J.-P. \& Stork A.L., 1997. Enumération des plantes à fleurs d'Afrique Tropicale. Gamopétales: Ericaceae à Lamiaceae. Génève (Suisse): CJB; 712 pp.

Midgley G., Clayton Y.M. \& Hay J.R., 1998. Atlas de poche de mycologie. Paris (France):
Médecine-Science; $154 \mathrm{pp}$.

Tatsadjieu L.N., Essia Ngang J.J., Ngassoum M.B. \& Etoab F.-X., 2003. Antibacterial and antifungal activity of Xylopia aethiopica, Monodora myristica, Zanthoxylum xanthoxyloýdes and Zanthoxylum leprieurii from Cameroon. Fitoterapia 74: 469-472.

Tra Bi F.H., 1997. Utilisations des plantes, par l'homme, dans les forêts classées du HautSassandra et de Scio, en Côte d'Ivoire. Thèse 3ème cycle. Abidjan (Côte d'Ivoire): Université d'Abidjan-Cocody; 215 pp.

Vlietinck A.J., Van Hoof L., Totté J., Lasure A., Vanden Berghe D., Rwangabo P.C. \& Mvukiyumwami J., 1995. Screening of hundred Rwandese medicinal plants for antimicrobial and antiviral properties. J. Ethnopharmacol. 46: 31-47. 